\title{
Avaliação do Efeito do Extrato Etanólico de Piper umbellatum L. no Controle Alternativo de Rhizoctonia solani e Macrophomina phaseolina ${ }^{1}$
}

\section{Evaluation of the Ethanolic Extract Effect of Piper umbellatum L. in the Alternative Control of Rhizoctonia solani and Macrophomina phaseolina}

\author{
Danielle Emanuelle Gusmão*a; Patrícia Oliveira Chaves ${ }^{\mathrm{a}}$; Rosemary Matias Coelho ${ }^{\mathrm{a}}$; Denise Renata Pedrinho ${ }^{\mathrm{a}}$; \\ Silvia Rahe Pereira, Bianca Obes Corrêa ${ }^{a}$
}

\begin{abstract}
${ }^{a}$ Universidade Anhanguera Uniderp, Programa de Pós-Graduação Stricto Sensu em Produção e Gestão Agroindustrial. MS, Brasil.
*E-mail: danyegusmao@hotmail.com
\end{abstract}

\begin{abstract}
Resumo
Objetivou-se, neste trabalho, investigar o potencial do extrato da planta Piper umbellatum (Sin. Piper umbellata L.) no crescimento micelial de Rhizoctonia solani e Macrophomina phaseolina. Para avaliação das habilidades fungicida e ou fungistática, os fungos fitopatogênicos foram crescidos previamente em meio de cultura BDA (batata dextrose ágar), tendo sido realizado o teste de crescimento micelial, em meio de cultura BDA nas concentrações de 800, 1200, 1600, 2000 e $2400 \mu \mathrm{g} 100 \mathrm{~mL}^{-1}$. Foram vertidos $10 \mathrm{~mL}$ de meio das diferentes concentrações em placas de Petri, com quatro repetições para cada concentração. A avaliação do crescimento micelial para todos os patógenos foi realizada por medidas diárias do diâmetro das colônias. Na prospecção fitoquímica do extrato etanólico de $P$. umbellatum foi detectada a presença majoritária de compostos fenólicos (100\%), seguido dos flavonoides, esteroides e alcaloides (75\%), os taninos, triterpenos e açúcares redutores (50\%) e com menor frequência as saponinas $(25 \%)$. As concentrações testadas não inibiram o crescimento micelial dos fungos, no entanto se observou que houve interferência no desenvolvimento da colônia de $R$. solani, pois não foi observada a formação de micro escleródios, estruturas estas formadas na testemunha e em função da presença de metabólitos secundários no extrato que podem apresentar atividade antifúngica, é recomendável a continuação do estudo fitoquímico de $P$. umbellatum, para isolamento e identificação de substâncias responsáveis pela atividade e determinar suas frações ativas, visando o controle de agentes fitopatogênicos.
\end{abstract}

Palavras-chave: Plantas Fungicidas. Piperaceae. Estratos Vegetais. Rhizoctonia solani; Macrophomina phaseolina.

\begin{abstract}
The objective of this work was to investigate the potential of Piper umbellatum (Sin. Piper umbellata L.) plant extract on the mycelial growth of Rhizoctonia solani and Macrophomina phaseolina. To evaluate fungicidal and or fungistatic abilities, phytopathogenic fungi were previously grown in BDA culture medium (potato dextrose agar), mycelial growth test was performed in BDA culture medium at concentrations of 800 , 1200, 1600, 2000 and $2400 \mu \mathrm{g} 100 \mathrm{~mL}-1.10 \mathrm{~mL}$ of medium of the different concentrations were poured into Petri dishes, with four replicates for each concentration. Mycelial growth evaluation for all the pathogens was performed by daily measurements of colony diameter. Phytochemical prospecting of P. umbellatum ethanolic extract showed the presence of phenolic compounds (100\%), followed by flavonoids, steroids and alkaloids (75\%), tannins, triterpenes and reducing sugars (50\%) and less frequently. the saponins (25\%). The tested concentrations did not inhibit the mycelial fungi growth, however, it was observed that there was interference in the development of R. solani colony, as the formation of micro sclerodes, structures formed in the control was not observed and due to the presence of secondary metabolites. In the extract that may present antifungal activity, the continuation of the phytochemical study of P. umbellatum is recommended for isolation and identification of substances responsible for the activity and to determine their active fractions, aiming at the control of phytopathogenic agents.
\end{abstract}

Keywords: Fungicidal plants; Piperaceae; Plant extracts; Rhizoctonia solani; Macrophomina phaseolina.

\section{Introdução}

Para o auxílio no controle de doenças, o uso racional de agrotóxicos possui efeito positivo, entretanto, em longo prazo, além do surgimento de fitopatógenos resistentes às substâncias químicas, esses produtos trazem efeitos nocivos como degradação dos recursos naturais e a saúde humana (SCHWAN-ESTRADA et al., 2000).

Com isto, a preocupação da sociedade com o impacto das práticas agrícolas no ambiente e a contaminação com produtos fitossanitários vêm alterando o cenário agrícola, resultando na presença de segmentos de mercado, que visam aquisição de produtos alternativos, estimulando a implementação do manejo e o uso de substâncias bioativas (MORANDI; BETTIOL; 2008). Essas inquietações têm levado ao desenvolvimento de sistemas de cultivo mais sustentáveis e, na busca de suprir as necessidades dos consumidores e produtores no desejo da redução do uso de agrotóxicos, diversas alternativas vêm sendo estudadas e indicadas para o controle de doenças de plantas (FIGUEREDO et al., 2006).

Entre os principais patógenos habitantes de solo se destaca a Macrophomina phaseolina e Rhizoctonia solani patógenos estes que atacam diversas culturas, formam estruturas de resistência para sobrevivência nas entressafras (MICHEREFF et al., 2005).

A resistência destes fitopatógenos, entre outros, está ligada ao uso frequente e dosagens não indicadas para controle de doenças, além do uso de moléculas que têm abrangência sobre diferentes tipos de agentes fitopatogênicos (GARCIA et al., 2012). Dessa forma, se faz necessária a busca por 
métodos alternativos para o controle de doenças, tornando-se a utilização de produtos naturais, como extratos de espécies vegetais uma alternativa promissora (GONÇALVES et al., 2009; ANDRADE et al., 2017).

A diversidade de substâncias ativas, em plantas medicinais, tem motivado o desenvolvimento de pesquisas envolvendo o uso de extratos vegetais, no intuito de explorar suas propriedades fungitóxicas e podendo ser utilizadas diretamente pelo produtor ou servir de matéria-prima para síntese de novos fungicidas. A família Piperaceae apresenta vários exemplares com estas potencialidades acima exploradas, uma dessas é a espécie $P$. umbellatum L. (CELOTO et al., 2008; RODRIGUES et al., 2012).

Dessa forma, objetivou-se avaliar o potencial fungitóxico do extrato etanólico de P. umbellatum no crescimento micelial de $R$. solani e M. phaseolina.

\section{Material e Métodos}

\subsection{Coleta, obtenção do extrato de $P$. umbellatum e prospecção química}

As folhas de P. umbellatum foram obtidas de 10 matrizes, nas primeiras horas da manhã, na horta de Plantas Medicinais e Aromáticas da Universidade Anhanguera-Uniderp, Unidade Agrárias, Campo Grande, MS, Brasil (20²6’21” Sul e longitude 54³2’27’). Após a identificação, um espécime foi catalogado e incorporado à coleção da Universidade Uniderp.

As folhas frescas foram secas em estufa circuladora de ar a $45^{\circ} \mathrm{C}\left(\mathrm{MARCONI}^{\circledR}, \mathrm{MA} 35\right)$, durante 48 horas, trituradas em moinho elétrico $\left(\mathrm{MARCONI}^{\circledR}, \mathrm{MA048)}\right.$ ), tamisadas e o pó (500 g) foi extraído com etanol $(99,5 \%)$ em banho de ultrassom (Ultrasonic Cleaner ${ }^{\circledR}$ ) durante 60 minutos, seguido de extração por maceração estática, durante sete dias. $\mathrm{O}$ material foi filtrado e o solvente evaporado, obtendo-se o extrato etanólico bruto, o qual foi submetido à análise química.

A prospecção fitoquímica foi desenvolvida utilizando o extrato etanólico, por meio de reações de caracterização, avaliando-se a presença de compostos fenólicos, taninos, flavonoides, antocianinas, cumarinas, antraquinonas, alcaloides, esteroides, triterpenos, heterosideos cardiotônicos, heterosideos cianogênicos, saponinas e açúcares redutores, segundo metodologia adaptada de Simões et al. (2017). Os resultados foram classificados, como: reação negativa (-), discreto (turvação) $( \pm)$, fracamente positiva $(+)$, positiva parcial $(+ \pm)$, positiva $(++)$, fortemente positiva $(++ \pm)$ e, alta intensidade $(+++)$, com frequência de $0,5,15,25,50,75$ e $100 \%$, respectivamente.

Os grupos químicos majoritários do extrato extrato etanólico foram confirmados pela análise no espectro na região UV-visível $\left(\right.$ Femto $\left.^{\circledR}, 800 \mathrm{XI}\right)$, com a determinação do comprimento de onda de máxima absorbância, na faixa de 200 a $800 \mathrm{~nm}$. O etanol foi utilizado como branco e os espectros foram comparados com dados da literatura (JURASEKOVA et al., 2006; KASAL et al., 2010; SILVERSTEIN et al.,
2014). As análises confirmatórias foram desenvolvidas com três repetições.

\subsection{Determinação dos compostos fenólicos, flavonoides, Taninos Condensados e alcaloides}

Do extrato etanólico $100 \mathrm{mg}$ foram utilizados para determinação dos teores de fenóis (FT). O ácido gálico (10 a $300 \mu \mathrm{g} \mathrm{mL}^{-1}$ ) foi empregado como padrão para construção da curva de calibração ( $\left.\mathrm{y}=0,0077 \mathrm{x}-0,0228 ; \mathrm{R}^{2}=0,9985\right)$. Os testes foram realizados sendo utilizado um espectrofotômetro na região de $750 \mathrm{~nm}$, cubetas de quartzo seguindo o Método Folin-Ciocalteu's (SOUSA et al., 2007).

Para quantificação dos flavonoides no extrato etanólico foi utilizado o método adaptado por Peixoto et al. (2008) e como padrão quercetina $\left(0,5 \mathrm{mg} \mathrm{mL}^{-1}\right)$ para construir a curva de calibração nas concentrações de: 0,$04 ; 0,2 ; 0,4 ; 2 ; 4 ; 8 ; 12 ; 16$; $20 \mu \mathrm{g} \mathrm{mL}^{-1}\left(\mathrm{y}=0,0637 \mathrm{x}-0,0067 \mathrm{R}^{2}=0,9991\right)$. As análises foram realizadas por espectrofotometria no comprimento de onda de $420 \mathrm{~nm}$, em cubetas de quartzo.

Os teores de taninos condensados foram determinados misturados $1 \mathrm{mg}$ do extrato etanólico em uma solução de hidrometanólica (MeOH: $\mathrm{H}_{2} \mathrm{O}: 80: 20 \mathrm{v}: \mathrm{v}$ ) e adicionado $5 \mathrm{~mL}$ de vanilina ácida ( $8 \%$ de $\mathrm{HCl}$ aquoso concentrado e $4 \%$ de vanilina em metanol). A catequina foi usada como padrão para construção da curva padrão e como branco o metanol. As misturas foram incubadas em banho-maria por 20 minutos, ao atingir a temperatura ambiente se fez a leitura em 510 nm (BROADHURST; JONES, 1978). Os resultados foram expressos como equivalentes de catequina em mg por $100 \mathrm{~g}$ de extrato.

O delineamento experimental foi de três repetições para cada concentração e o cálculo das médias foi acompanhado do desvio padrão.

A quantificação dos alcaloides totais foi desenvolvida utilizando $40 \mathrm{~mL}$ do extrato etanólico, na concentração de $1000 \mu \mathrm{g} / \mathrm{mL}$, posteriormente, acidificado para $\mathrm{pH}$ entre 2,0 - 2,5 com $\mathrm{HCl} 1 \mathrm{~mol} / \mathrm{L}$ e $4 \mathrm{~mL}$ de reagente Dragendorff e centrifugado a $2400 \mathrm{rpm} / 30$ minutos. O sobrenadante foi descartado e o resíduo tratado com uma solução contendo $1 \mathrm{~mL}$ de álcool etílicol; $2 \mathrm{~mL}$ de sulfito de sódio (1\%) e novamente centrifugados (2400 rpm/30 minutos). O sobrenadante foi descartado e o resíduo tratado com ácido nítrico concentrado ( $2 \mathrm{~mL}$ ). A solução foi transferida para um balão volumétrico de $50 \mathrm{~mL}$ e o volume completado com água destilada. Desta solução, uma alíquota $(1 \mathrm{~mL})$ foi utilizada e adicionado em $5 \mathrm{~mL}$ de tioureia a $3 \%(\mathrm{p} / \mathrm{v})$, homogeneizado e a leitura em espectrofotômetro em $435 \mathrm{~nm}$. A solução de ácido nítrico e tioureia foi usada como branco e como padrão a berberina a linearidade foi obtida entre 40 e $200 \mu \mathrm{g} / \mathrm{mL}$. O teor de alcaloides foi expresso em $\mathrm{mg} / 100 \mathrm{~g}$ de peso seco do extrato (SREEVIDYA; MEHROTRA, 2003). 


\subsection{Obtenção dos isolados fitopatogênicos}

Os isolados fitopatogênicos que foram utilizados no presente trabalho são provenientes da coleção de Microorganismos Fitopatogênicos do Laboratório de Fitopatologia da Universidade Anhanguera-Uniderp, Campo Grande, MS e oriundos de plantas de soja com sintomas típicos das doenças provocadas por estes patógenos, isolados no ano de 2017, que foram inseridos na plataforma do SISGen, para obtenção da autorização e registro de acesso ao patrimônio genético do Conselho de Gestão do Patrimônio Genético.

\subsection{Atividade fungistática}

Para avaliação das habilidades fungistáticas do extrato vegetal de $P$. umbellatum, os fungos fitopatogênicos foram crescidos previamente em meio de cultura BDA (BatataDextrose-Ágar), apresentando sete dias de crescimento no dia do início do ensaio.

Para o teste de crescimento micelial, que foi realizado para todos os patógenos, foi preparada uma solução estoque, contendo 0,2 g do extrato etanólico de P. umbellatum em 100 $\mathrm{mL}$ de etanol $(99,5 \%)$. A partir desta solução foram utilizadas alíquotas de 0,$8 ; 1,2 ; 1,6 ; 2,0$ e 2,4 mL, separadamente vertidas, em meio de cultura BDA fundente, até completar o volume de $100 \mathrm{~mL}$, no qual foram obtidas as concentrações de $800,1200,1600,2000$ e $2400 \mu \mathrm{g} 100 \mathrm{~mL}^{-1}$. Como controle foram preparadas duas soluções, uma contendo etanol $(99,5 \%)$ adicionado ao meio BDA (2400 $\left.\mu \mathrm{g} 100 \mathrm{~mL}^{-1}\right)$, denominada solução etanólica e outra somente com o meio BDA (sem o extrato), a testemunha.

Foram vertidos $10 \mathrm{~mL}$ de meio das diferentes concentrações em placas de Petri, com quatro repetições para cada concentração. Após a solidificação do meio, foi depositado no centro de cada placa, um disco de $0,5 \mathrm{~cm}$ de diâmetro do micélio dos fungos, em ensaios separados, previamente repicados sete dias ao procedimento. Posteriormente, as placas foram vedadas com papel filme, e mantidas em câmara de crescimento, a $25^{\circ} \mathrm{C}$, com fotoperíodo de 12 horas.

A avaliação do crescimento micelial para todos os patógenos foi realizada por medidas diárias do diâmetro das colônias, obtida pela média de duas medidas diametralmente opostas, ocorrendo até que a testemunha alcançasse os bordos da placa.

O delineamento experimental utilizado foi do tipo inteiramente casualizado, com quatro repetições para cada uma das concentrações do extrato etanólico de $P$. umbellatum, a testemunha foi constituída de placas contendo meio de cultura sem adição de extrato.

\section{Resultados e Discussão}

Na prospecção fitoquímica do extrato etanólico de $P$. umbellatum foi detectada a presença majoritária de compostos fenólicos (100\%), seguido dos flavonoides, esteroides e alcaloides $(75 \%)$, os taninos, triterpenos e açúcares redutores
$(50 \%)$ e, com menor frequência $(25 \%)$, as saponinas (Figura $1)$.

Figura 1 - Frequência (\%) das classes de metabólitos secundários do extrato etanólico das folhas de P. umbellatum, Campo Grande, MS.

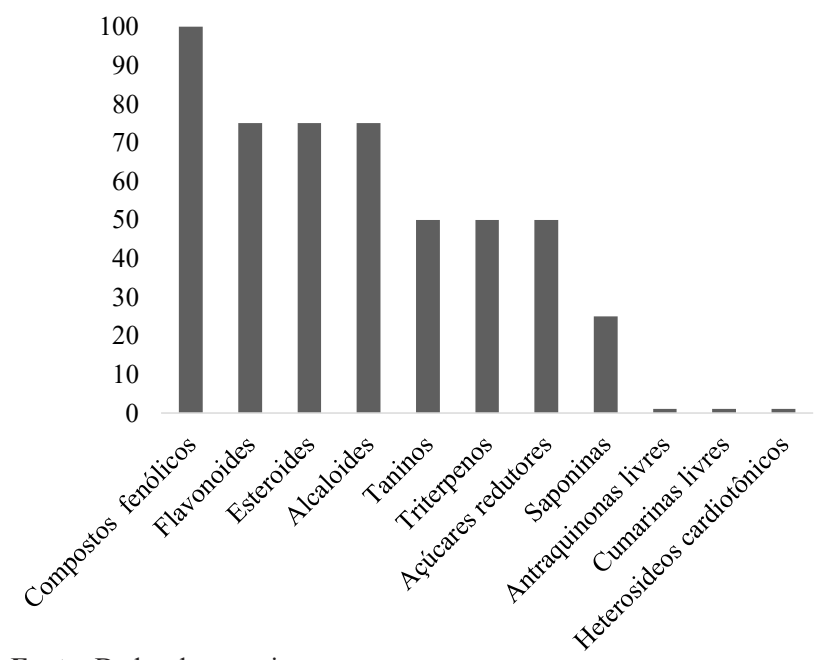

Fonte: Dados da pesquisa.

Para P. umbellatum foram identificados na planta cerca de 50 compostos pertencentes aos fenois simples e derivados (ciclo-hexano oxigenado, hidroquinonas, ácido benzoico), polifenois (flavonoides, lignanas, neolignanas), terpenos, amidas e alcaloides (PARMAR et al., 1997; TABOPDA et al., 2008, SILVA et al., 2014), o que está de acordo com os fitoconstituintes apresentados na Figura 1 e Quadro 1.

Quadro 1 - Comprimento de onda máximo na região visível por UVvisível do extrato etanólico de Piper umbellatum, Campo Grande, Brasil

\begin{tabular}{|c|c|c|}
\hline $\begin{array}{c}\text { Classe de } \\
\text { Metabólitos } \\
\text { Predominantes }\end{array}$ & $\lambda_{\max (\mathrm{mm})}$ & $\begin{array}{c}\text { Dados da } \\
\text { Literatura* }\end{array}$ \\
\hline $\begin{array}{c}\text { Compostos } \\
\text { Fenólicos }\end{array}$ & $260,0 \mathrm{~nm}$ & Entre $220-290(1)$ \\
\hline \multirow{2}{*}{ Flavonoides } & Entre $270-285 \mathrm{~nm}$ & $\begin{array}{c}\text { Entre } 240-285 \\
\mathrm{~nm}(2)\end{array}$ \\
\cline { 2 - 3 } & Entre $320-385 \mathrm{~nm}$ & $\begin{array}{c}\text { Entre } 300-400 \mathrm{~nm} \\
(2)\end{array}$ \\
\hline Esteroides & $\begin{array}{c}\text { Entre } 210 \mathrm{~nm} \text { e } 220 \\
\mathrm{~nm}\end{array}$ & $\begin{array}{c}\text { entre } 206 \mathrm{~nm} \text { e } 220 \\
\mathrm{~nm}(3)\end{array}$ \\
\hline Alcaloides & $193 \mathrm{~nm}$ & $194 \mathrm{~nm}$ \\
$297 \mathrm{~nm}$ & $220,380 \mathrm{~nm}$ \\
\hline
\end{tabular}

Fonte: Dados da pesquisa.

A caracterização do extrato etanólico e a confirmação das classes de metabólitos secundários, evidenciadas na prospecção fitoquímica, ocorreu também por espectrofotometria no UV/ visível (Quadro 1),

Foi possível verificar a existência de similaridades entre o screennig fitoquímico e as bandas máximas de absorção dos constituintes majoritários, os compostos fenólicos (100\%), flavonoides $(75 \%)$ e alcaloides $(75 \%)$. O espectro UV mostra uma forte absorção em $260,0 \mathrm{~nm}$, característico de compostos fenólicos (Quadro 1). O espectro se estende além dos 385 
nm, região que compreende a banda de absorção UV de flavonoides (ZUANAZZI et al., 2017).

É possível observar, no espectro de UvVisível 2 bandas de absorção, uma entre 270 - 285 nm ( $\lambda$ máx. $\left._{{ }_{\mathrm{MeOH}}}\right)$, na forma de um ombro, e uma segunda banda entre 320 - 385 nm ( $\lambda$ máx. $\mathrm{MeOH}$ ), característico de flavonoides (Quadro 1). Em geral, os flavonoides apresentam bandas entre 240 - $285 \mathrm{~nm}$ (Banda II), correspondente à absorção do anel A (hemicetal) e uma segunda banda entre 300 - $400 \mathrm{~nm}$ (Banda I), que representa a absorção do anel B (ZUANAZZI et al., 2010). O perfil das moléculas individuais de flavonoides depende do número de grupos hidroxilas presentes e sua localização (JURASEKOVA et al., 2006).

A banda de absorção entre $206 \mathrm{~nm}$ e $220 \mathrm{~nm}$ observada no espectro para o extrato etanólico é comum para esteroides (Quadro 1), um fitohormônio com estrutura básica formada de 17 átomos de carbono, dispostos em quatro anéis ligados entre si, essa estrutura química é denominada de Ciclo-pentanoperidro-fenantreno (Anéis A, B e C), os quais estão ligados a um anel ciclopentano (Anel D) (PÉREZ; ESCANDAR, 2013). Porém, dependendo do sistema de conjugação de dieno (homo ou heteroanular) com a presença do sistema enona, caracteres hexocíclicos de ligações duplas e outras conjugações adicionais, entre outras na molécula de esterol, seu espectro pode apresentar uma absorção máxima por exemplo entre 220 e $350 \mathrm{~nm}$ (KASAL et al., 2010).

Os achados fotoquímicos são confirmados com os teores de compostos fenólicos, flavonoides, taninos e alcaloides que podem ser considerados elevados (Quadro 2). Investigações fitoquímicas com gênero Piper (família Piperaceae), que inclui um grande número de espécies, conhecidas popularmente como Pariparoba, elucidaram vários compostos de classes típicas, tais como: amidas, terpenos, derivados do ácido benzoico, carotenos, hidroquinonas, lignanas, neolignanas e alguns alcaloides (REGASINI et al., 2008, REGASINI et al., 2009; COTINGUIBA et al., 2009).

Quadro 2 - Resultados Perfil químico (UV visível), da quantificação de fenóis totais e flavonoides do extrato etanólico das folhas de Piper umbellatum, Campo Grande, MS

\begin{tabular}{|c|c|c|c|c|}
\hline Extr. & $\begin{array}{c}\text { Fen. Tot. } \\
\left(\mathbf{m g ~ g}^{-1}\right)\end{array}$ & $\begin{array}{c}\text { Flav. } \\
\left(\mathbf{m g ~ g}^{-1}\right)\end{array}$ & $\begin{array}{c}\text { Tan. } \\
\left(\mathbf{m g ~ g}^{-1}\right)\end{array}$ & $\begin{array}{c}\text { Alcaloides } \\
\left(\mathbf{m g ~ g}^{-1}\right)\end{array}$ \\
\hline \multirow{2}{*}{ Etanólico } & $325,86 \pm$ & $199,51 \pm$ & $55,45 \pm$ & $78,75 \pm$ \\
& 0,82 & 0,132 & 2,09 & 2,55 \\
\hline
\end{tabular}

*Fenois totais (mg de EAG g ${ }^{-1} \pm$ DP). Flavonoides (mg de Querc. $\mathrm{g}^{-1} \pm$ DP). Taninos Condensados (mg de cat. $\mathrm{g}^{-1} \pm \mathrm{DP}$ ). Alcaloides (mg de berberina $\mathrm{g}^{-1} \pm \mathrm{SD}$. * Mean $\pm \mathrm{SD}$.

Fonte: Dados da pesquisa.

Os constituintes típicos ainda do gênero Piper estão os alcaloides e amidas, a maioria dos compostos conhecidos são as piperidinas, pirrolidinas ou isobutilaminas e tem como o principal representante a piperina. Para P. umbellatum, a classe citada são N-benzoilmescalina (ISOBE et al., 2002), piperumbelactanas (TABOPDA et al., 2008), este último pertence ao grupo das piperolactamas, não são comuns, mas espécies do Gênero, sendo classificadas como aporfinoides, uma vez que seus esqueletos são semelhantes aos alcaloides aporfínicos (KUMAR et al., 2003). As piperolactamas possuem absorção em UvVisível $(\mathrm{MeOH})$ entre $194 \mathrm{~nm}$ e $380 \mathrm{~nm}$, valores similares também encontrados no extrato etanólico das folhas de P. umbellatum, uma banda intensa em 193 nm e uma segunda banda em 297nm.

$\mathrm{O}$ extrato etanólico de $P$. umbelatum apresentou percentuais expressivos de fenóis totais $325,86 \pm 0,82 \mathrm{mg}$ de ácido gálico/100g $\left(\mathrm{Y}=0,078 \mathrm{x}-0,031\right.$ fenois $\left.\mathrm{R}^{2}=0,9959\right) \mathrm{em}$ quantidades superiores a outras espécies do Gênero, como para as três variedades de Piper betel os valores obtidos mostraram teores entre $820,53 \pm 10,8$ e 72,25 $\pm 1,42 \boldsymbol{\mu g}$ de ácido gálico/g (RATHEE et al., 2006), os teores de fenóis totais também foram inferiores para Piper cubeba $(123,1 \pm 0,05 \mu \mathrm{g}$ de ácido gálico/g) e Piper nigrum (62,3 $\pm 0,08 \mu \mathrm{g}$ de ácido gálico/g) (NAHAK; SAHU, 2011), assim como para as folhas de Piper nigrum 0,36 mg ácido gálico/mL GAE (SAPAM et al., 2018).

Para os teores de flavonoides totais, os valores obtidos foram de $199,51 \pm 0,132 \mathrm{mg}$ de quercetina/100g e foram superiores aos obtidos por Silva Junior et al. (2014), 165mg quercetina/g $\left(\mathrm{Y}=0,132 \mathrm{x}+0,0353 \mathrm{R}^{2}=0,9949\right)$, para as folhas de $P$. umbellatum. Não foram encontrados valores para os taninos nas fontes pesquisadas, porém podem ser considerados expressivos em relação aos teores de compostos fenólicos totais e flavonoides.

Os resultados dos teores de fenóis e flavonoides obtidos para a espécie em estudo e em comparação para outras do gênero Piper demonstram uma variabilidade dos teores de fenóis e flavonoides, o que estimula a continuidade de estudos fitoquímicos com esta espécie.

O extrato etanólico de $P$. umbellatum possui predominância de polifenóis e derivados (fenólicos e flavonoides). Os flavonoides, glicoflavonoides, terpeno glicosilado e lignanas foram isolados das folhas da planta em estudo (BALDOQUI et al., 2009). Esses grupos apresentam, em sua estrutura, o grupo carboxila $(\mathrm{OH})$, que têm a capacidade de se ligar a receptores enzimáticos de micro-organismos, inibindo a síntese de proteínas e favorecem modificações metabólicas de patógenos, inibindo a síntese de ácidos nucléicos durante a formação da membrana citoplasmática e o metabolismo energético. Enquanto os triterpenos têm um esqueleto lipofílico básico, o que facilita a quebra da membrana celular e com sua parte polar dentro da célula, esses atuam desabilitando os receptores residuais de aminoácidos de fungos (CUSHNIE; LAMB, 2005).

Os metabólicos secundários produzidos pelas plantas têm sido objeto de estudos e por apresentarem atividade antimicrobiana se mostram promissores para o controle de fitopatógenos (BALBI-PEÑA et al., 2006). Esses compostos provocam a interrupção na membrana plasmática, inibindo a ação das enzimas fúngicas, ocasionando desorganização no conteúdo celular de diferentes espécies de fungos (SCHWANESTRADA et al., 2000). 
Da mesma forma, os compostos fenólicos e taninos são considerados inibidores de enzimas hidrolíticas produzidas por patógenos e responsáveis pela resistência dos tecidos vegetais. Assim como os flavonoides e taninos também atuam como elicitores e/ou mecanismos químicos de defesa contra vírus, bactérias e fungos sua toxicidade depende da quantidade de grupos metilados e do número de substituintes na formação do anel aromático, o que os torna mais tóxicos, interferindo na constituição das membranas celulares de fungos (ANDRADE et al., 2017).

Os resultados dos teores de fenóis e flavonoides obtidos para a espécie em estudo e em comparação para outras do gênero Piper demonstraram variabilidade dos teores de fenóis e flavonoides, o que estimula a continuidade de estudos fitoquímicos com esta espécie.

Para o teste in vitro se observa no Quadro 3, que as concentrações testadas não proporcionaram inibição do crescimento micelial do fungo M. phaseolina, já para $R$. solani se observa que a concentração de $1200 \mu \mathrm{g} / 100 \mathrm{~mL}$ do extrato etanólico de $P$. umbellatum reduziu o crescimento micelial deste fungo, em relação à testemunha e outras concentrações testadas. No entanto, após o término das avaliações com o fungo $R$. solani, em todas as concentrações do extrato não houve observação de micro escleródios, quando comparados à testemunha, que apresentou início de formação destas estruturas dois dias após o término das avaliações do ensaio in vitro. Para o fungo $M$. phaseolina não foram observadas peculiaridades com relação à morfologia da colônia.

Quadro 3 - Crescimento micelial de $M$. phaseolina e $R$. solan sob diferentes concentrações do extrato etanólico de folhas de $P$. umbellatum

\begin{tabular}{|c|c|c|}
\hline \multicolumn{3}{|c|}{ Crescimento micelial $(\mathrm{mm})$} \\
\hline Tratamentos & M. phaseolina & R. solani \\
\hline Testemunha & $2.57 \mathrm{a}^{*}$ & $2.25 \mathrm{a}$ \\
\hline $800 \mu \mathrm{g} / 100 \mathrm{~mL}$ & $2.60 \mathrm{a}$ & $2.31 \mathrm{~b}$ \\
\hline 1200 & $2.60 \mathrm{a}$ & $2.19 \mathrm{c}$ \\
\hline 1600 & $2.54 \mathrm{a}$ & $2.30 \mathrm{~b}$ \\
\hline 2000 & $2.57 \mathrm{a}$ & $2.35 \mathrm{~b}$ \\
\hline 2400 & $2.52 \mathrm{a}$ & $2.34 \mathrm{~b}$ \\
\hline
\end{tabular}

*Médias seguidas pela mesma letra minúsculas na coluna não diferem significativamente entre si segundo Tukey $(5 \%)$.

Fonte: Dados da pesquisa.

Avaliações in vitro sobre o crescimento micelial de fungos, em relação às diferentes concentrações de extratos vegetais, são usados para selecionar e reduzir o número de tratamentos que poderão ser testados in vivo, no entanto, as concentrações testadas in vitro não proporcionam o controle esperado. As concentrações testadas no presente trabalho estão entre as testadas e indicadas para fitopatógenos, variando de 125 a $3000 \mu \mathrm{g} / 100 \mathrm{~mL}$, conforme metodologias de diferentes autores (ARAÚJO et al., 2014; BRITO; NASCIMENTO, 2015; PANSERA et al., 2015; SOUSA et al., 2018).

Para as concentrações 800, 1600, 2000 e $2400 \mu \mathrm{g} / 100$ $\mathrm{mL}$, nota-se efeito estimulador para o fungo $R$. solani. É possível levantar a hipótese de que embora os alcaloides sejam produtos naturais típicos de plantas, e por apresentarem nitrogênio em sua cadeia, é possível haver interferência no processo, uma vez que o elemento químico nitrogênio é essencial para constituição molecular e celular de microorganismos (SANTOS et al., 2011).

Além disso, no gênero Piper é comum a presença de microorganismos endofíticos que podem produzir toxinas e alterar a composição dos metabólicos secundários, aumentando a taxa de alcaloides das plantas, aumentando a quantidade de açúcares e outros metabólitos, que ao invés de interferirem negativamente no crescimento micelial, podem estimular o crescimento fúngico (SANTOS; VARAVALLO, 2011).

Esta pode ser uma das interferências observadas sobre as concentrações testadas, uma vez que não foi observada redução dos patógenos. Sangeetha e Rawal (2008) relatam que meios com elevadas concentrações de açúcar favorecem o desenvolvimento dos fungos e monossacarídeos por serem uma fonte de carbono para os fungos, um componente importante para o crescimento e esporulação está relacionado ao seu processo de nutrição.

Este fato fica comprovado pela avaliação de açúcares redutores do extrato, com frequência de $50 \%$, o que comprova que a presença destes como fonte de carbono pode ter influenciado diretamente o crescimento micelial dos fitopatógenos e, dependendo do tipo de açúcar há influência positiva no crescimetno micelial, como já foi relatado por Andrade et al., (2017). Segundo Sangeetha e Rawal (2008), os monossacarídeos são fonte de carbono para fungos, componente essencial para seu crescimento e esporulação, uma vez que estão relacionados ao seu processo de nutrição.

Uma perspectiva para aplicação dos resultados deste trabalho está na elaboração de produtos fitossanitários naturais para o manejo integrado de doenças de plantas. Em função da presença de metabólitos, que podem apresentar atividade antifúngica, é recomendável a continuação do estudo fitoquímico de $P$. umbellatum, para isolamento e identificação de substâncias responsáveis pela atividade e determinar suas frações ativas. Contudo, novas pesquisas devem ser realizadas, tendo em vista que tais estudos ainda são escassos, visando investigação fitoquímica de extratos vegetais, no controle de agentes fitopatogênicos.

\section{Conclusão}

O extrato etanólico de $P$. umbellatum testado teve efeito fungistático in vitro sobre o crescimento micelial do fungo $R$. solani.

Foram identificados onze metabólitos secundários (açúcares redutores, taninos, esteroides, antraquinonas, heterosideos cardiotônicos, cumarinas, triterpenos, compostos fenólicos, saponinas, alcaloides e flavonoides). 


\section{Referências}

ANDRADE, B. S. et al. Fitoquímica, potencial antioxidante e antifúngico de Byrsonima crassifolia no controle de fitopatógenos de solo. Braz. J. Biology, v.78, n.1, 2017. doi: 10.1590/15196984.166532

ARAÚJO, E. R. et al. Extratos de Piper marginatum e Azadirachta indica no controle de Colletotrichum scovillei em pimentão. Pesq. Agropec. Bras., v.49, n.49, p.88-94, 2014.

BALDOQUI, D.C. et al. Flavonas, lignanas e terpeno de Piper umbellata (Piperaceae). Química Nova, v.32, n.5, p.1107- 1109, 2009.

BALBI-PEÑA, M. I. et al. Controle de Alternaria solani em tomateiro por extratos de Curcuma longa e curcumina - I. avaliação in vitro. Fitopatol. Bras., v.31, n.3, p.310-314, 2006. doi: 10.1590/S0100-41582006000400012.

BRITO, N.M.; NASCIMENTO, L.C. Potencial fungitóxico de extratos vegetais sobre Curvularia eragrostidis (P. Henn.) Meyer in vitro. Rev. Bras. Plantas Med., v.17, n.2, p.230-238, 2015. doi: 10.1590/1983-084X/10_057.

BROADHURST, R. B.; JONES, W. T. Analysis of condensed tannins using acidified vanillin. J. Scie. Food Agricul., v.29, n.9, p.788-794, 1978. doi: 10.1002/jsfa.2740290908.

CELOTO, M. I. B. et al. Atividade antifúngica de extratos de plantas a Colletotrichum gloeosporioides. Acta Scie. Agronomy, v.30, n.1, p.1-5, 2008. doi: 10.4025/actasciagron.v30i1.1104.

COTINGUIBA, F. et al. Piperamides and their derivatives aspotential antitrypanosomal agents. Med. Chem. Res., v.19, n. 1B, 2009. doi: 10.1007/s00044-008-9161-9.

CUSHNIE, T.P.; LAMB, A.J. Antimicrobial activity of flavonoids. Int. J. Antim. Agents, v.26, n.5, p. 343-356, 2005. doi: 10.1016/j. ijantimicag.2005.09.002.

FIGUEREDO, M.L.C.; MARTINS-DIAS, A.M.P; CRUZ, I. Relação entre a lagarta-do-cartucho e seus agentes de controle biológico natural na produção de milho. Pesq. Agropec. Bras., v. 41, n. 12 , p.1693-1698, 2006

GARCIA, A. et al. Atividade antifúngica de óleo e extratos vegetais sobre Sclerotinia sclerotiorum. Riccely, v. 28, n.1, p.48$57,2012$.

GONÇALVES G.G.; MATTOS L. P. V.; MORAIS LAS. Óleos essenciais e extratos vegetais no controle de fitopatógenos de grãos de soja. Horticul. Bras., v.27, p.S102-S107, 2009.

ISOBE, T.; OSAKI, A.; NAGATA, K. Antibacterial constituents aganist helicobacter pylori of Bras. Med. Plant, v.122, n. 4, p.291-294, 2002.

JURASEKOVA, Z. et al. Surface-enhanced Raman scattering of flavonoids. J. Raman Spectroscopy, v.37, n.11, p.1239-1241, 2006. doi: 10.1002/jrs.1634.

KASAL, A.; BUDESINSKY, M.; GRIFFITHS, W. J. Spectroscopic methods of steroid analysis. In: Steroid analysis, 2010. p. 27-161.

KUMAR, S. Spectroscopy of organic compounds. New Delhi: New Age International, blishers, 2006.

KUMAR, V.; PRASAD, A. K.; PARMAR, V. S. Naturally occurring aristolactams, aristolochic acids and dioxoaporphines and their biological activities. Nat. Prod. Reports, v.20, n.6, p.565-583, 2003. doi: 10.1039/B303648K.

MICHEREFF，S.J.; ANDRADE，D.E.G.T.; MENEZES，M. Ecologia e manejo de patógenos radiculares em solos tropicais. Recife: UFRPE, 2005.
MORANDI, M. A. B.; BETTIOL, W. Integração de métodos biocompatíveis no manejo de doenças e pragas: experiências em plantas ornamentais e medicinais. Trop. Plant Pathol., v.33, p.3134, 2008.

NAHAK, G.; SAHU, R. K. Phytochemical evaluation and antioxidant activity of Piper cubeba and Piper nigrum. J. Appl. Pharm. Scie; v.1, n.8, p.153, 2011.

PANSERA, M. R. et al. Utilização de tinturas vegetais e óleo essencial no controle do mofo-branco em alface. Rev. Bras. Agroecol., v.10, p.87-91, 2015.

PARMAR, V. S. et al. Fitoquímica do gênero Piper. Elsevier Scie., v.46, n. 4, p.597-673, 1997.

PÉREZ, R. L.; ESCANDAR, G. M. Spectrofluorimetric study of estrogen-cyclodextrin inclusion complexes in aqueous systems. Analyst, v.138, n.4, p.1239-1248, 2013.

PEIXOTO SOBRINHO, T. J. S. et al. Validação de metodologia espectrofotométrica para quantificação dos flavonóides de Bauhinia cheilantha (Bongard) Steudel. Rev. Bras. Ciênc. Farm., n. 44,p. 683-689, 2008. doi: 10.1590/S1516-93322008000400015.

RATHEE, J. S. et al. Antioxidant activity of Piper betel leaf extract and its constituents. J. Agricul. Food Chem., v. 54, n. 24, 9046-9054, 2006. doi: 10.1021/jf061679e.

REGASINI, L. O. et al. Atividade tripanocida de Piper arboreum e Piper tuberculatum (Piperaceae). Rev. Bras. Farm., v.19, p.199203, 2009.

REGASINI, L. O. et al. Radical Scavenging Capacity of Piper arboreum and Piper tuberculatum (Piperaceae). Latin Am. J. Pharm., v.27, n.6, p.900-903, 2008.

RODRIGUES, E. et al. Avaliação da atividade antifúngica de extratos de gengibre e eucalipto in vitro e em fibras de bananeira infectadas com Helminthosporium sp. Acta Scie. Agron., v.28, n. 1, p.123-127, 2006.

RODRIGUES, E. R. et al. Pothomorphe umbellate: antifungal activity against strains of Trichophyton rubrum. J. Mycol. Méd., v.22, p.265-269, 2012. doi: 10.1016/j.mycmed.2012.05.005.

SANGEETHA, C. G.; RAWAL, R. D. Nutritional Studies of Colletotrichum gloeosporioides (Penz.) Penz. and Sacc. the incitant of mango anthracnose. World J. Agricul. Scie., v.4, n.6, p.717-720, 2008.

SANTOS, J. O. et al. Influência de diferentes fontes de nitrogênio no crescimento e na produção de lipases pelo fungo Fusarium oxysporum ATCC 48112 por fermentação submersa. In: REUNIÃO ANUAL DA SBPC, 63, 2011, Anais eletrônicos. Disponível em: <http://www.sbpcnet.org.br/livro/63ra/resumos/ resumos/5742.htm>. Acesso em: 13 out. 2019.

SANTOS, T.T.; VARAVALLO, M. A. Aplicação de microrganismos endofíticos na agricultura e na produção de substâncias de interesse econômico. Semina Ciênc. Biol. Saúde, v.32, n.2, p.199, 2011. doi: 10.5433/1679-0367.2011v32n2p199.

SAPAM, R. et al. Screening of phytochemicals and determination of total phenolic content, anti-oxidant and antimicrobial activity of methanolic extract of Piper nigrum leaves. Pharm. Res., v.8, n.2, p.1354-1360, 2018.

SCHWAN-ESTRADA, K.R.F.; STANGARLIN, J.R.; CRUZ, M.E.S. Uso de extratos vegetais no controle de fungos fitopatogênicos. Rev. Floresta, v.30, n.12, p.129-137, 2000. doi: 10.5380/rf.v30i12.2361.

SIMÕES, C. M. O. et al. Farmacognosia: do produto natural ao medicamento. Porto Alegre: Artmed, 2017.

SILVA JUNIOR, I. F. et al. Evaluation of acute toxicity, 
antibacterial activity, and mode of action of the hydroethanolic extract of Piper umbellatum L. J. Ethnopharmacol., v.151, n.1, p.137-143, 2014. doi: 10.1016/j.jep.2013.10.011.

SILVERSTEIN, R.M; WEBSTER, F.X. Identificação espectrométrica de compostos orgânicos. Química Nova. LCT: Rio de Janeiro, 2014.

SOUSA, I. et al. Atividade fungitóxica de extratos de Piper sp. Contra os fungos Rhizoctonia solani e Sclerotium rolfsii. Rev. Am. Ciênc. Amb., v.9, n.3, p.50-56, 2018. doi: 10.6008/CBPC21796858.2018.003.0005.

SOUSA, C. M. et al. Fenóis totais e atividade antioxidante de cinco plantas medicinais. Quim. Nova, n.30, p.351-355, 2007. doi: 10.1590/s0100-40422007000200021.

SREEVIDYA, N.; MEHROTRA, S. Spectrophotometric method for estimation of alkaloids precipitable with dragendorff's reagent in plant materials. J. AOAC Int., v.86, n.6, p.1124-1127, 2003.

TABOPDA, T. K. et al. Bioactive aristolactams from Piper umbellatum. Phytochemistry, v. 69, n. 8, p. 1726-1731, 2008. doi: 10.1016/j.phytochem.2008.02.018.

ZUANAZZI, J.A.S.; MONTANHA, J.A. Flavonoides. In: SIMÕES, C.M.O. et al. Farmacognosia: da planta ao medicamento. Porto Alegre, 2010. p.577-614. 